\title{
Cyclin-dependent kinase 5 regulates the proliferation, motility and invasiveness of lung cancer cells through its effects on cytoskeletal remodeling
}

\author{
JUN-LI LIU ${ }^{1 *}$, RUN-XIA GU ${ }^{1 *}$, XIAO-SHU ZHOU ${ }^{1}$, FANG-ZHENG ZHOU ${ }^{2}$ and GANG WU ${ }^{1}$ \\ ${ }^{1}$ Cancer Center, Union Hospital, Tongji Medical College, Huazhong University of Science and Technology, Wuhan, \\ Hubei 430022; ${ }^{2}$ Department of Oncology, Suizhou Central Hospital, Suizhou, Hubei 441300, P.R. China
}

Received August 21, 2014; Accepted May 1, 2015

DOI: $10.3892 / \mathrm{mmr} .2015 .3868$

\begin{abstract}
Determining the molecular phenotype is a key to understanding and predicting the metastatic potential and the prognosis for patients with lung cancer. Our previous study demonstrated that increased expression of cyclin-dependent kinase 5 (CDK5) in patients with non-small cell lung cancer (NSCLC) is associated with a poorer prognosis. The present study aimed to further investigate the underlying mechanism of CDK5 in vitro and in vivo using the A549 human NSCLC cell line. A 3-(4,5-dimethylthiazol-2-yl)-2,5-diphenyltetrazolium bromide assay was used to quantify the proliferation of the A549 cells; migration assay and invasiveness assays were performed using Transwell chambers and wound healing assays were used to assess cell motility, which was assessed by measuring the movement of cells. Inhibition of CDK5 by roscovitine and small interfering (si)RNA was used to investigate the mechanism of CDK5 in the process of A549 lung cancer cell proliferation, migration and invasion. The results demonstrated that functional inhibition of CDK5 using roscovitine and siRNA markedly suppressed the proliferation of A549 cells and resulted in a reduced tumor mass in vivo. In addition, the hinhibition of CDK5 reduced the migration and invasiveness of the A549 cells in vitro and in vivo. Notably, CDK5 inhibition also impaired tumor cell cytoskeletal remodeling and led to loss of cell polarity, which may partially explain the reduction of A549 cell mobility and invasiveness.
\end{abstract}

Correspondence to: Professor Gang Wu, Cancer Center, Union Hospital, Tongji Medical College, Huazhong University of Science and Technology, 156 Wu Jia Dun, Wuhan, Hubei 430022, P.R. China E-mail: xhzlwg@163.com

*Contributed equally

Abbreviations: CDK5, cyclin-dependent kinase 5; NSCLC, non-small cell lung cancer; PFS, progression-free survival rate

Key words: cyclin-dependent kinase 5, lung cancer, invasiveness, motility, roscovitine, small interfering RNA
The results of the present study revealed that CDK5 may be important in the regulation of migration and invasiveness in NSCLC through its effects on cytoskeletal remodeling.

\section{Introduction}

Lung cancer has long been the leading cause of cancer-associated mortality worldwide (1). Although numerous therapeutic strategies have improved significantly, the presence of locally advanced or metastatic lung cancer reduces the curability of this life-threatening disease. Several efforts have been made to understand the mechanism and molecular pathogenesis of this disease, with promise in the development of more effective targeted treatment strategies for lung cancer, particularly non-small cell lung cancer (NSCLC). Certain small molecular inhibitors have been developed against certain tyrosine kinases, including epidermal growth factor receptor and vascular endothelial growth factor receptor (2); however, the overall progression free survival rate has not improved satisfactorily (3). The poor survival rate is predominantly attributed to aggressive growth, advanced stage at diagnosis and local recurrence (4). The identification of effectors, which modulate growth, cell migration and invasion will assist in understanding the process of disease progression and enable the development of novel therapeutic targets for patients with NSCLC.

Cyclin-dependent kinase 5 (CDK5) has been recognized as a key factor in regulating the migration and plasticity of neuron cells $(5,6)$. Compared with other CDKs, CDK5 is considered to be an essential regulator of neuronal differentiation, rather than a cell cycle regulator; and controls a wide range of functions, including synapse formation, neuronal migration and axon guidance (7), all of which indicate that CDK5 is important as a 'migration mediator'. Notably, CDK5 had been found to be correlated with increased invasion in prostate carcinoma, glioblastoma multiforme $(8,9)$, pancreatic cancer cells $(10,11)$ and medullary thyroid cancer (12). The inhibition of the activity of CDK5 decreases cancer cell migration and invasion (13), however, the mechanism underlying the involvement of CDK5 in tumorigenesis remains to be fully elucidated. Our previous study demonstrated that the overexpression of CDK5 is closely correlated with reduced survival rates in patients 
with NSCLC (14), however, its intracellular mechanisms, linking the activity of CDK5 with the progression of cancer remain to be determined.

The present study investigated the impact of CDK5 on the proliferation, migration and invasiveness of the A549 human NSCLC cell line, by using its functional inhibitor, roscovitine, and CDK5 small interfering (si)RNA, to attempt to elucidate its underlying molecular mechanisms.

\section{Materials and methods}

Materials. The materials and reagents used in the present study were purchase from the following providers. Roscovitine was purchased from Sigma-Aldrich (St Louis, MO, USA); Matrigel basement membrane matrix and collagen type 1 were obtained from Becton Dickinson (Bedford, MA, USA); primary rabbit anti-CDK5 polyclonal (sc-173; $1: 1,000)$ and rabbit anti- $\beta$-actin polyclonal (sc-130656; 1:2,000) antibodies were purchased from Santa Cruz Biotechnology, Inc. (Santa Cruz, CA, USA); anti-rabbit immunoglobulin (Ig) $\mathrm{G}$ alkaline phosphatase-conjugated antibody was purchased from Bio-Rad Laboratories, Inc. (Munich, Germany); RPMI 1640 medium and fetal bovine serum (FBS) were purchased from Hyclone (Logan, UT, USA); fluorescein isothiocyanate (FITC)-conjugated phalloidin was obtained from Sigma-Aldrich. All other reagents were of the purest grade available. The current study was approved by the Ethics Committee of Union Hospital, Tongji Medical College, Huazhong University of Science and Technology (Wuhan, China).

Cdk5 kinase activity analysis and CDK5 gene silencing. The A549 human lung adenocarcinoma cell line was purchased from American Type Culture Collection (Manassas, VA, USA). The cells were maintained in RPMI-1640 medium supplemented with $10 \%$ fetal bovine serum, and incubated at $37^{\circ} \mathrm{C}$ in a humidified atmosphere containing $5 \% \mathrm{CO}_{2}$.

A549 cells stably transfected with non-specific siRNA and siRNA-cdk5 were purchased from GenePharm (Shanghai, China). The target sequence (TGAAGTGTAACCCTGTCC A) was used to downregulate CDK5 in vitro. A non-silencing siRNA sequence (target sequence 5-AATTCTCCGAACGTG TCACGT-3) was used as a negative control.

Analysis of Cdk5 kinase activity was performed using a GENMED in vitro Cdk5 Kinase Activity kit (GENMED Scientifics, Inc., Arlington, MA, USA), according to the manufacturer's instructions.

Cell proliferation, invasion and migration assays. A 3-(4,5-dimethylthiazol-2-yl)-2,5-diphenyltetrazolium bromide (MTT) assay was used to quantify the proliferation of the cells. The A549 cells were seeded into 96-well plates at density of 3,000 cells in $0.2 \mathrm{ml}$ medium. Roscovitine was used at a range of concentrations $(0,5,10$ and $20 \mu \mathrm{mol} / \mathrm{l})$. Duplicate plates contained six replicate wells per assay. Following culture in 5\% $\mathrm{CO}_{2}$ at $37^{\circ} \mathrm{C}$ for 12,24 or $72 \mathrm{~h}, 20 \mu \mathrm{MTT}(5 \mathrm{mg} / \mathrm{ml})$ was added to each well for $4 \mathrm{~h}$ at $37^{\circ} \mathrm{C}$ to allow MTT to form formazan crystals, which were solubilized in dimethyl sulfoxide. The optical density (OD) was measured using a microplate reader (3500; Bio-Rad Laboratories, Inc.) at $490 \mathrm{~nm}$. The percentage of cell growth was calculated by comparing the OD at A490 between the treated and untreated control cells. The same procedure was performed on the A549 cells stably transfected with non-specific siRNA and siRNA-cdk5.

The migration assay was performed using Transwell chambers, containing $6.5 \mathrm{~mm}$ diameter polycarbonate filters (8 $\mu \mathrm{m}$ pore size) coated with $50 \mu \mathrm{l}$ Matrigel. The A549 cells were adjusted to a density of $2 \times 10^{5}$ cells $/ \mathrm{ml}$ in serum-free RPMI-1640 medium. The cell suspension (200 $\mu \mathrm{l})$ was added into the upper chamber, and $500 \mu \mathrm{l}$ RPMI-1640 medium, supplemented with $10 \%$ FBS, was loaded in the lower chamber. Roscovitine $(0,5,10$ or $20 \mu \mathrm{mol} / \mathrm{l})$ was added to the upper chamber. Following culture in $5 \% \mathrm{CO}_{2}$ at $37^{\circ} \mathrm{C}$ for $24 \mathrm{~h}$, the cells, which had migrated through the filters were fixed with 100\% cold methanol (Wuhan Boster Biological Engineering Co., Ltd., Wuhan, China), stained with hematoxylin (Wuhan Boster Biological Engineering Co., Ltd.) and were counted under a light microscope (DC6-163; National Optical \& Scientific Instruments, Inc., Schertz, TX, USA). The migration assay was also performed using Transwell chambers, without Matrigel, but using the same procedures as for the invasion assay. The same assays were performed on the A549 cells stably transfected with non-specific siRNA and siRNA-cdk5.

Reverse transcription-quantitative polymerase chain reaction (RT-qPCR). Total RNA was extracted using TRIzol reagent (Invitrogen Life Technologies, Carlsbad, CA, USA). The purity of the RNA (A260/A280>1.8) was examined using a spectrophotometer (UV7502; Xinmao Co., Ltd., Shanghai, China), and then the RNA $(1 \mu \mathrm{g})$ was used to synthesize cDNA using the SuperScript First-Strand Synthesis kit(Thermo Fisher Scientific, Pittsburgh, PA, USA) following the manufacturer's instructions. RT-qPCR was then performed using the SYBR Green qPCR Super Mixture (Takara Bio, Inc., Shiga, Japan) and an ABI Prism 7500 Sequence Detection system (Applied Biosystems Life Technologies, Foster City, CA, USA). All samples were run in triplicate in each experiment. The following primer sequences were used: Human Cdk5, forward 5'-CGATGACCA GTTGAAGAGGAT-3' and reverse 5'-TCTGGCAGCTTGGTC ATAGA-3'; human GAPDH, forward 5'-GAAGGTGAAGGT CGGAGTC-3' and reverse 5'-ATGGGATTTCCATTGATG AC-3'.

Wound healing assay. In the wound healing assays, cell motility was assessed by measuring the movement of cells across a wound scratch. The monolayer was gently and slowly scratched with a new $1 \mathrm{ml}$ pipette tip across the center of the well. The long-axial of the tip was kept perpendicular to the bottom of the well at all times. The speed of the wound closure was monitored after 12,18 and $24 \mathrm{~h}$ by measuring the ratio of the distance of the wound at $0 \mathrm{~h}$. Each experiment was performed in triplicate.

Western blotting. The cells $\left(1 \times 10^{6}\right)$ were lysed in cell lysis buffer and whole-cell lysates. The protein concentrations were determined using a bicinchoninic acid protein assay reagent kit (Pierce Biotechnology, Inc., Rockford, IL, USA). The proteins (30 $\mu \mathrm{g}$ /lane; $30 \mu \mathrm{g} / 30 \mu \mathrm{l}$ ) were separated on $10 \%$ sodium dodecyl sulfate-polyacrylamide gels and electrotransferred onto polyvinylidene difluoride membranes (Wuhan Boster Biological Engineering Co., Ltd.). The membranes were blocked with blocking buffer (5\% bovine serum albumin in 
phosphate-buffered saline with $0.1 \%$ Tween 20 ) for $1 \mathrm{~h}$ at room temperature, probed with primary antibodies overnight at $4^{\circ} \mathrm{C}$, incubated with secondary antibodies for $1 \mathrm{~h}$ at room temperature, and developed using an ECL Advance ${ }^{\mathrm{TM}}$ western blotting detection kit (GE Healthcare Life Sciences, Chalfont, UK).

Xenografted tumor growth and numbers of metastatic tumors in the lungs of nude mice. Cultured A549 cells (5x106 cells per mouse), stably transfected with non-specific siRNA and siRNA-cdk5 were subcutaneously injected into the back of 4-week old female BALB/c athymic nude mice $(n=3$ for each group) that were housed individually at $23 \pm 1^{\circ} \mathrm{C}$ with a $12 \mathrm{~h} / 12 \mathrm{~h}$ light/dark cycle. Food and water were available ad libitum and the mice were anesthetized with inhaled isoflurane (Abbott Laboratories, North Chicago, IL, USA). Mice were sacrificed by $\mathrm{CO}_{2}$ inhalation followed by cervical dislocation. Tumor volumes $\left(\mathrm{mm}^{3}\right)$ were determined once each week using calipers and the following formula: (width ${ }^{2} \mathrm{x}$ height)/ 2 . After 5 weeks, the mice were sacrificed and the tumors were resected.

Cultured A549cells ( $1 \times 10^{7}$ cells per mouse) stably transfected with non-specific siRNA or siRNA-cdk5 were subcutaneously injected into the caudal vein of 4-week old female BALB/c athymic nude mice ( $n=3$ for each group). After 5 weeks, the mice were sacrificed and the number of metastatic tumors in the lungs were counted.

Visualization using laser confocal microscopy and scanning electron microscopy. The A549 cells $\left(1 \times 10^{6}\right)$ were treated with $20 \mu \mathrm{mol} / 1$ roscovitine for $48 \mathrm{~h}$ at $37^{\circ} \mathrm{C}$. The cells were then fixed with $4 \%$ formaldehyde for $20 \mathrm{~min}$ at $37^{\circ} \mathrm{C}$ and the cells were treated with $0.2 \%$ Triton $\mathrm{X}-100$ at room temperature for $10 \mathrm{~min}$, following which FITC-conjugated phalloidin $(10 \mu \mathrm{g} / \mathrm{ml})$ was added to stain the cells for $1 \mathrm{~h}$ at $37^{\circ} \mathrm{C}$. The cells were counterstained with 4',6-diamidino-2-phenylindole (Wuhan Boster Biological Engineering Co., Ltd.) for $10 \mathrm{~min}$ at $37^{\circ} \mathrm{C}$, and slides were mounted using $50 \%$ glycerol. Cell morphology was then examined under a laser confocal microscope.

The A549 cells were cultured on collagen gel plaques for $48 \mathrm{~h}$, and were then fixed using 1\% osmium tetroxide (Wuhan Boster Biological Engineering Co., Ltd.) for $1.5 \mathrm{~h}$ at $37^{\circ} \mathrm{C}$. Following dehydration using an alcohol gradient, the samples were immobilized with a conductive adhesive (Atom Adhesives, Fort Lauderdale, FL, USA) and sprayed with carbon and gold (Wuhan Boster Biological Engineering Co., Ltd.). The samples were observed under a scanning electron microscope and images were captured.

Statistical analysis. The results are expressed as the mean \pm standard deviation. Statistical differences among the groups were determined using a two-tailed Student's t-test. Statistical analyses were performed using SPSS 11.0 software (SPSS, Inc., Chicago, IL, USA). P $<0.05$ were considered to indicate a statistically significant difference.

\section{Results}

Roscovitine and siRNA markedly inhibit CDK5 activity and suppress A549 lung cancer cell growth. To investigate the role of CDK5 in NSCLC, the present study first determined its effect on A549 cell proliferation. CDK5 kinase activity was observed in the A549 cell lines and this activity was inhibited by roscovitine at concentrations between 5 and $40 \mu \mathrm{M}$ (Fig. 1A). The expression of CDK5 in the A549 cell lines was also analyzed using western blotting, which was suppressed by roscovitine (Fig. 1B). As shown in Fig. 1C, roscovitine at low concentrations had no significant inhibitory effect on A549 cell proliferation until $40 \mu \mathrm{M}$. Accordingly, $40 \mu \mathrm{M}$ roscovitine was identified as an effective suppressive concentration. To exclude the possibility that the inhibition of the proliferation of A549 cells was due to the drug toxicity of roscovitine, the apoptotic status was determined in the presence of various concentrations of roscovitine. To further confirm the above results in vivo, lenti-CDK5-siRNA was transfected into A549 cells, which demonstrated that CDK5 siRNA was effective at reducing the mRNA and protein expression levels of CDK5, compared with the untreated cells. The control siRNA had no effect (Fig. 1D and E).

Furthermore, the lenti-CDK5-siRNA A549 cells and counterpart control cells were injected subcutaneously in BALB/c nude-mice for the analysis of tumor growth. The mice were sacrificed and the size of the subcutaneous xenografts were determined after 35 days. As shown in Fig. 1F, silencing of CDK5 significantly reduced the tumor growth of the A549 cells in vivo, thus corroborating the finding that CDK5 regulated A549 cell proliferation.

CDK5 inhibition by roscovitine and siRNA inhibits the motility and migration of A549 lung cancer cells through the effects on the cytoskeleton and microvilli remodeling. CDK5 is reported to be associated with neuronal migration (7). In this regard, the present study investigated whether A549 tumor cell migration was also regulated by CDK 5 kinase by using a Transwell assay for cell migration on the A549 cell, to determine whether the motility of the cells was dependent on CDK5 activity. The A549 cells were cultured in the presence of various concentrations of roscovitine in the upper chamber of a Transwell for $24 \mathrm{~h}$. A dose-dependent inhibitory effect on migration was observed, which peaked at a concentration of $20 \mu \mathrm{mol} / 1$ roscovitine (Fig. 2A).

siRNA knockdown of the expression of CDK5 was also effective in reducing the motility of the A549 lung cancer cells. The number of A549 cells transfected with lenti-CDK5-siRNA in the chamber was $\sim 30 \%$ of the number of control siRNA cells (Fig. 2B).

To further support the above results, a wound healing assay for cell migration was performed using the lenti-CDK5-siRNA A549 cells. As shown in Fig. 2C, the cells treated with CDK5 siRNA exhibited significantly impaired repopulating abilities across the wound and impaired migrating capabilities at different time points, particularly at $24 \mathrm{~h}$, suggesting the importance of CDK5 in the migration of A549 cells.

Since remodeling of the cytoskeleton and microvilli are responsible forcell motility andmigration, the present study subsequently investigated whether the inhibition of CDK5 attenuated cell motility and migration through impairment of cytoskeletal and microvilli remodeling. Fluorescence-conjugated phalloidin was used to detect the cytoskeleton in the roscovitine-treated or untreated A549 cells. Laser confocal microscopy revealed a compact and directional alignment with fibrous tension in the control group, whereas F-actin was aligned and aggregated 
A
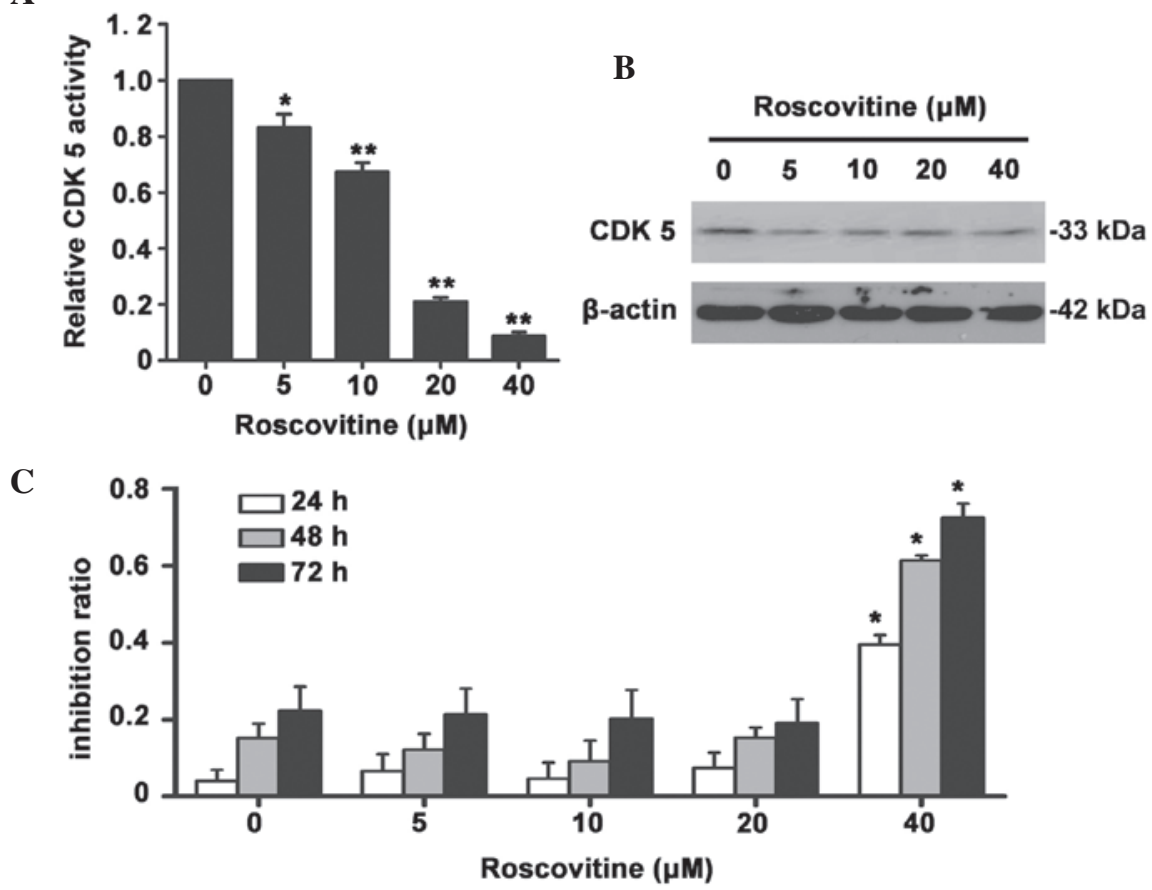

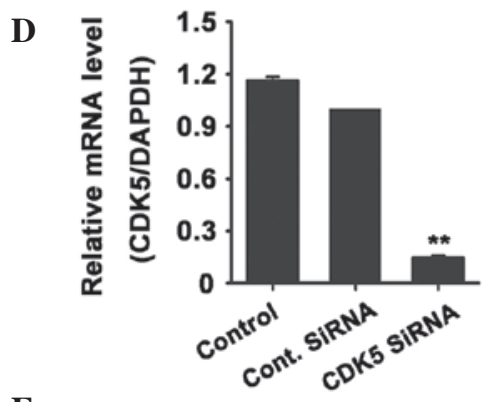

$\mathbf{F}$

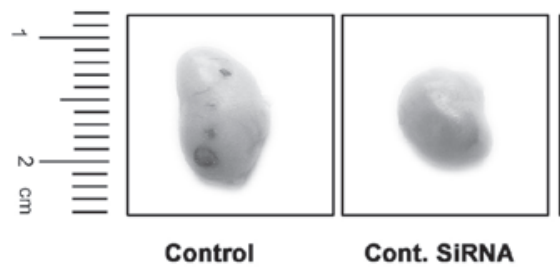

$\mathbf{E}$
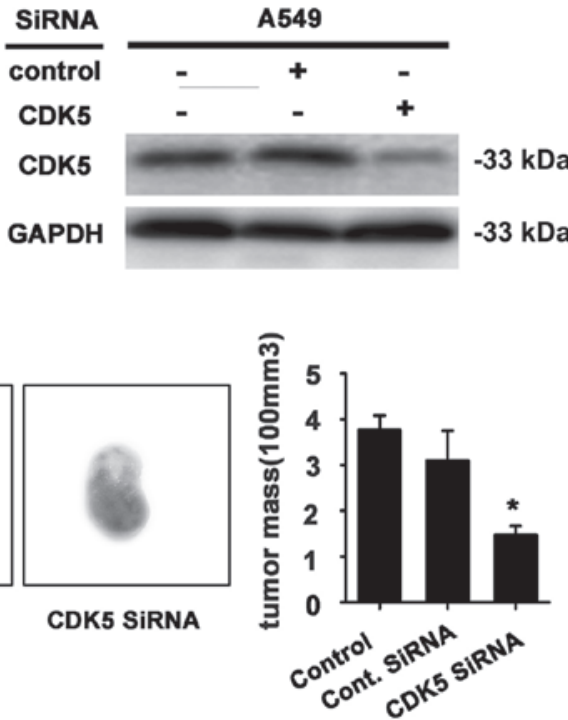

Figure 1. Inhibition of CDK5 suppresses lung cell growth in vitro and ex vivo. (A) Kinase assay of CDK5 from A549 non small cell lung cancer cell lysates. Concentration-dependent inhibition of CDK5 activity was observed and maximal functional inhibition of CDK5 on the A549 cells was reached at a roscovitine concentration of $40 \mu \mathrm{mol} / 1$. (B) A549 cells were treated with the indicated concentration of roscovitine for $24 \mathrm{~h}$, and the protein expression of CDK5 level was determined using western blot analysis and corrected by $\beta$-actin. (C) A 3-(4,5-dimethylthiazol-2-yl)-2,5-diphenyltetrazolium bromide assay was performed to determine the effects of roscovitine $(0,10,20$ and $40 \mu \mathrm{mol} / \mathrm{l})$ on the proliferation of A549 cells at $24 \mathrm{~h}, 48 \mathrm{~h}$ and $72 \mathrm{~h}$. (D and E) lenti-CDK5-siRNA and its counterpart control siRNA were transfected into A549 cells, and the mRNA and protein levels of CDK5 were measured using (D) reverse-transcription-quantitative polymerase chain reaction and (E) western blot analysis. (F) A549 cells transfected with non-specific siRNA, siRNA-CDK5 or blank A549 cells were inoculated subcutaneously into the right flank of BALB/c nude mice, which were subsequently sacrificed after 35 days and the tumor masses were measured. Representative images of the tumors (left) and quantitative measurements (right) are shown, respectively. Data are representative of three independent experiments and are expressed as the mean \pm standard deviation. ${ }^{*} \mathrm{P}<0.05$ and ${ }^{* *} \mathrm{P}<0.01$, compared with $0 \mu \mathrm{M}$ roscovitine or the control siRNA. CDK5, cylcin-dependent kinase 5; siRNA, small interfering RNA; Cont, control.

in the $20 \mu \mathrm{mol} / 1$ roscovitine treatment group, with loss of the fibrous and directional characteristics of F-actin (Fig. 3A). These results indicated that roscovitine caused disruption of the cytoskeletal structure of the A549 lung cancer cells. In addition, further investigation of cell morphology using a scanning electron microscope revealed that the roscovitine-treated A549 cells exhibited impaired pseudopod formation and loss of cellular polarity, compared with control group (Fig. 3B).

Taken together, the results of roscovitine and siRNA treatment indicated that CDK5 was important in the motility and migration of these lung cancer cells through its effects on the cytoskeleton and microvilli remodeling. 
A

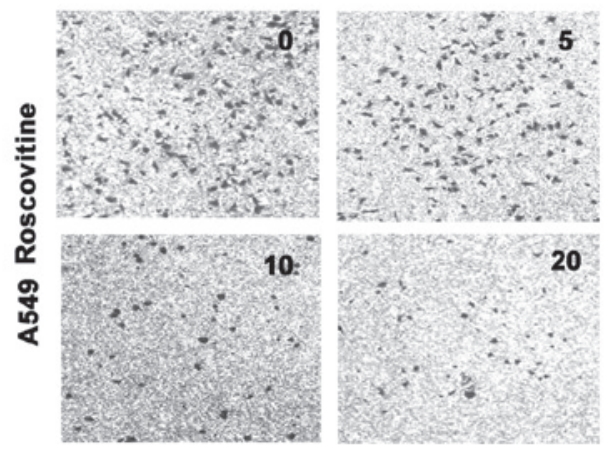

C
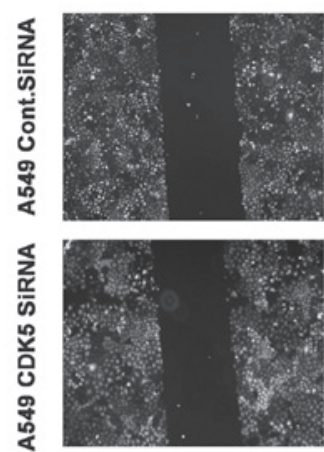

$\mathbf{O h}$
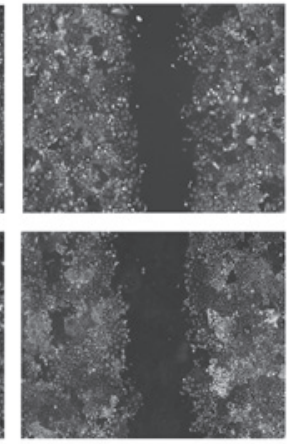

$12 \mathrm{~h}$
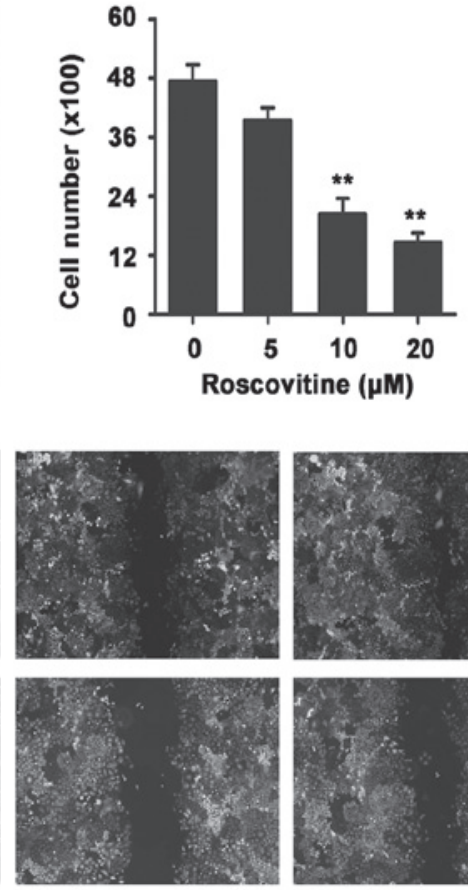

$18 \mathrm{~h}$
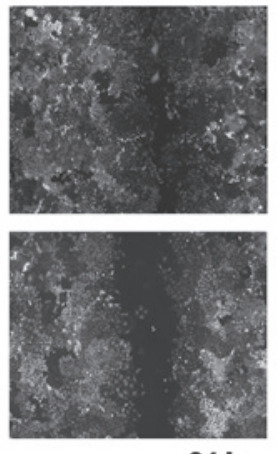

B
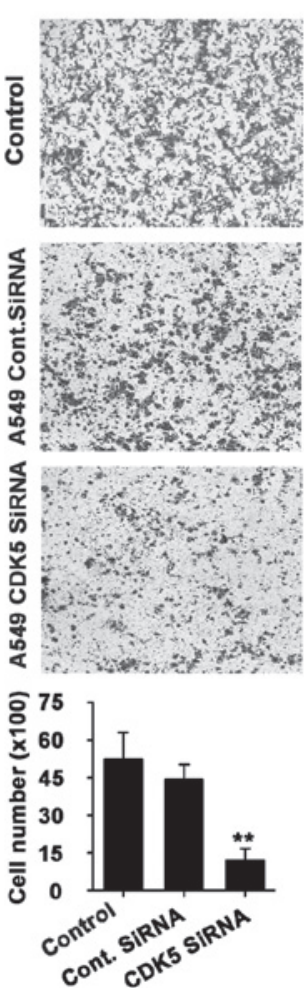

Figure 2. Functional inhibition of CDK5 decreases the motility and migration of A549 cells in vitro. (A) A549 cells were seeded into Transwell chambers with polycarbonate filters $(8 \mu \mathrm{m}$ diameter). Roscovitine was added, at the indicated concentrations, to the upper chambers. The number of cells was calculated at $24 \mathrm{~h}$. (B) Migration assay for A549 lung cancer cells transfected with non-specific siRNA or siRNA-cdk5, or blank A549 cells. Cell migration was calculated at $24 \mathrm{~h}$. (C) In vitro wound assay of the A549 cells transfected with siRNA-cdk5 at $0,12,18$ and $24 \mathrm{~h}$. ${ }^{*} \mathrm{P}<0.05$ and ${ }^{* *} \mathrm{P}<0.01$, compared with the $0 \mu \mathrm{M}$ roscovitine or the control. CDK5, cyclin-dependent kinase 5; siRNA, small interfering RNA; Cont, control.

A
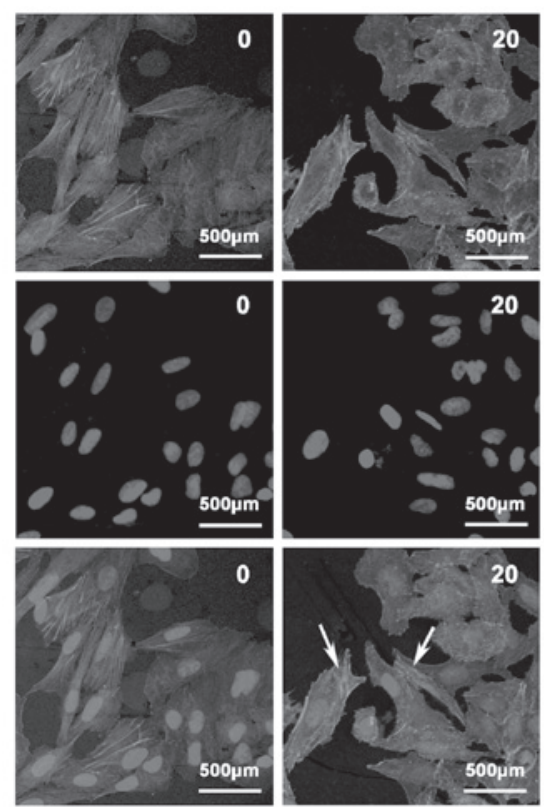

A549 Control

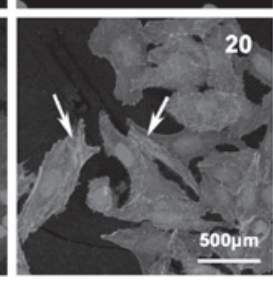

A549 Roscovitine
B

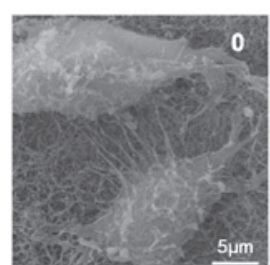

A549 Control

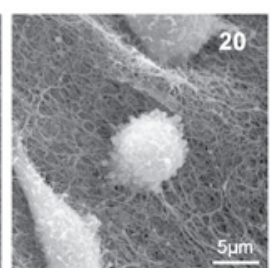

A549 Roscovitine

Figure 3. Inhibition of CDK5 impairs cytoskeletal remodeling and microfilament formation of lung cancer cells. (A) Immunofluorescent staining of F-actin in A549 cells in the presence of $20 \mu \mathrm{mol} / 1$ roscovitine. Counterstaining was performed using 4',6-diamidino-2-phenylindole. (B) Electron scanning microscopy revealed the detailed morphology of the A549 cells treated with or without roscovitine. CDK5, cyclin-dependent kinase 5.

CDK5 inhibition attenuates the invasiveness of lung cancer cells. As the decreased cell motility in lung cancer is considered to be correlated with lower invasive potential, the effects of
CDK5 inhibition by roscovitine and siRNA on the invasiveness of tumor cells were also investigated in the present study, in addition to the cell motility and migration. The invasive 
A

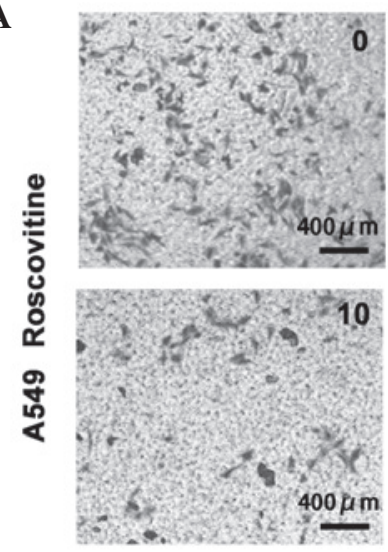

C

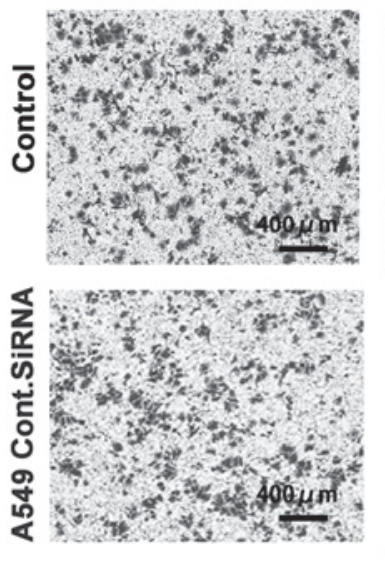

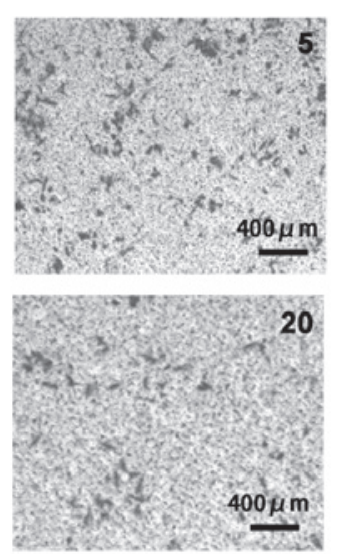
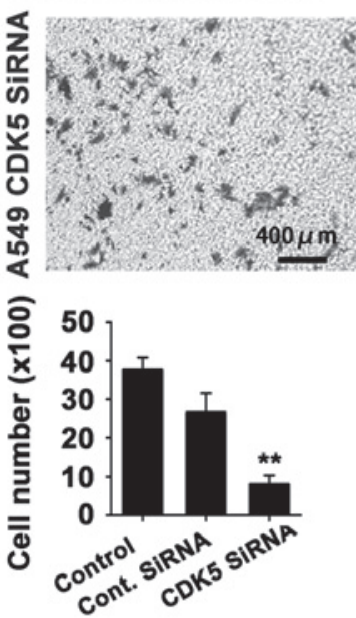

D

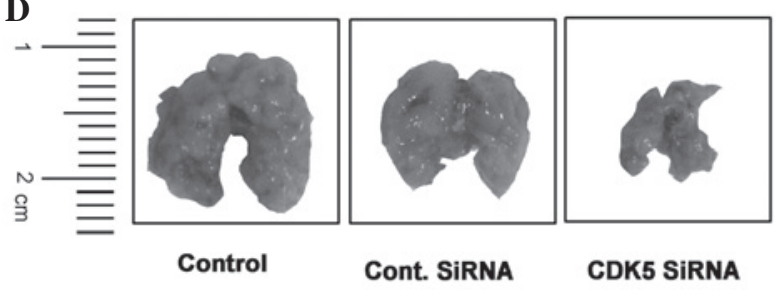

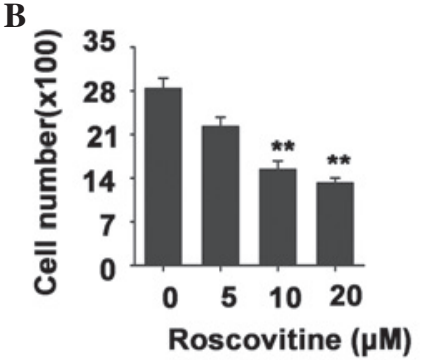

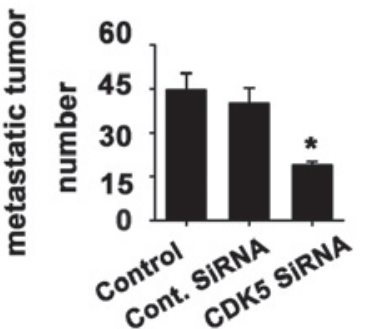

Figure 4. Inhibition of CDK5 attenuates the invasiveness of A549 cells in vitro and in vivo. (A) A549 cells were treated with roscovitine at the indicated concentrations for $48 \mathrm{~h}$ to inhibit CDK5 activity. The invasive ability of each group was measured using Transwell chambers with polycarbonate filters $(8 \mu \mathrm{m}$ pore size) coated with Matrigel. The images $0,5,10$ and 20 are representative of the groups treated with $0,5,10$ and $20 \mu$ mol/l roscovitine, respectively. (B) Quantification of the numbers of roscovitine-treated A549 cells, which passed through the Matrigel. (C) In vitro invasive assay of A549 cells treated with non-specific siRNA or siRNA-cdk5. (D) A549 cells transfected with non-specific siRNA or siRNA-cdk5 were injected into the tail vein of BALB/c nude mice for the analysis of pulmonary implantation. At 35 days post-tumor injection, the mice were sacrificed and implantation number in the lung was calculated. ${ }^{*} \mathrm{P}<0.05$, compared with the $0 \mu \mathrm{M}$ roscovitine or the control. CDK5, cyclin-dependent kinase 5; siRNA, small interfering RNA; Cont, control.

potential of the A549 lung cancer cells was determined using Matrigel-coated invasion chambers. The cells were cultured on the Matrigel-coated membrane in the presence of various concentrations of roscovitine. The invasiveness assay revealed that roscovitine markedly suppressed the invasiveness of the hA549 cells, and the invasion capacity of the tumor cells in the $20 \mu \mathrm{mol} / 1$ roscovitine group became $<50 \%$ of that of the control group (Fig. 4A and B). Furthermore, the lenti-CDK5-siRNA A549 cells also demonstrated decreased invasiveness (Fig. 4C). The CDK5 dominant-negative A549 cells invasion was only $\sim 30 \%$ of that of the empty vector control cells.

As the results of the present study demonstrated the importance of CDK5 in the motility and invasion of lung cancer cells, it was hypothesized that inhibiting the activity of CDK5 interferes with metastasis in vivo. To examine the role of the activity of CDK5 in metastasis, the present study performed in vivo spontaneous metastasis experiments using the A549 lung cancer cells. Subsequently, the lenti-CDK5-siRNA A549 cells and its counterpart control cells were injected, respectively, into the mice through the tail vein to analyze the invasiveness in vivo. At 35 days post-injection, the mice were sacrificed and lung surface metastases were evaluated. As shown in Fig. 4D, the invasion of cells was markedly reduced in the lenti-CDK5-siRNA A549 cells, indicating that CDK5 effectively regulated the invasiveness of the A549 cells.

\section{Discussion}

The high mortality rate for NSCLC may be attributed to its early metastasis and rapid local invasion (15). In the present 
study, CDK5 activity was observed to be important for NSCLC cell proliferation, migration and invasiveness, and the inhibition of CDK5 by roscovitine and siRNA markedly inhibited lung cancer cell growth and invasiveness, indicating CDK5 as a potential therapeutic target in NSCLC.

Previously, CDK5 was found to be uniquely involved in the development of the central nervous system, as an upstream central control element for neuronal migration. However, the involvement of the biological functions of CDK5 have diversified into cancer $(10,16)$. In our previous study $(14)$, Cdk5 and/or p35 were commonly expressed in two-thirds of NSCLC cases and its high expression level was indicative of an unfavorable prognosis in patients with NSCLC, which suggested novel concepts for use as a biomarker or for the development of molecular-targeted treatments for NSCLC. Demelash et al (10) extended these observations to lung neuroendocrine carcinoma, including small cell lung carcinoma and carcinoid. However, the functional role of CDK5 activity in cell proliferation, migration and invasiveness of lung cancer cells remains to be elucidated. The results of the present study confirmed that CDK5 mediated the motility and invasiveness of lung cancer cells in vitro and in vivo, and CDK5 inhibition via treatment of the lung cancer cells with roscovitine resulted in a reduction in the migration and invasion of the lung cancer cells in vitro, similar to results previously reported in prostate cancer (8). The specificity was confirmed by transfecting lung cancer cells with CDK5 siRNA, which led to decreased CDK5 activity and impaired migration. Of note, the results of the present study also confirmed that the inhibition of CDK5 affected cytoskeletal remodeling in the tumor cells. Previous studies have demonstrated that several molecules or receptors can induce cytoskeletal remodeling. Toll-like receptor 4 induces remodeling via phosphorylation of the myosin light chain for the release of microparticles, thus regulating tumor inflammatory microenvironment (17). Goodwin et al (18) reported that, in motor neuron cells, CDK-5 regulates microtubule orientation and in cancer, Strock et al (8) reported that inhibiting the activity of CDK5 resulted in changes in the microtubule cytoskeleton, loss of cellular polarity and loss of motility in prostate cancer. In the present study, inhibition of CDK5 resulted in failed microtubule assembly, thus leading to impaired mobility of the A549 lung cancer cells. In this regard, it is reasonable to suggest that CDK5 regulates the motility and migration of A549 cells, thus leading to the metastasis of lung cancer.

In conclusion, the results of the present study provided evidence that CDK5 is critical in promoting proliferation, invasiveness and metastasis in NSCLC and, thus, may be a potential therapeutic target that mediates cancer metastasis and invasiveness.

\section{Acknowledgements}

This study was supported by the National Natural Science Foundation of China (grant. no 81101766) to Dr Jun-Li Liu.

\section{References}

1. DeSantis C, Naishadham D and Jemal A: Cancer statistics for African Americans, 2013. CA Cancer J Clin 63: 151-166, 2013.

2. Mulligan LM: RET revisited: Expanding the oncogenic portfolio. Nat Rev Cancer 14: 173-186, 2014.

3. Tartour E and Zitvogel L: Lung cancer: Potential targets for immunotherapy. Lancet Respir Med 1: 551-563, 2013.

4. Albain KS, Crowley JJ, LeBlanc M and Livingston RB: Survival determinants in extensive-stage non-small-cell lung cancer: the Southwest Oncology Group experience. J Clin Oncol 9: 1618-1626, 1991.

5. Ghose A and Shashidhara LS: Cyclin beyond the cell cycle: new partners at the synapse. Dev Cell 21: 601-602, 2011.

6. McMinn PC: Recent advances in the molecular epidemiology and control of human enterovirus 71 infection. Curr Opin Virol 2: 199-205, 2012.

7. Dhavan R and Tsai LH: A decade of CDK5. Nat Rev Mol Cell Biol 2: 749-759, 2001.

8. Strock CJ, Park JI, Nakakura EK, Bova GS, Isaacs JT, Ball DW and Nelkin BD: Cyclin-dependent kinase 5 activity controls cell motility and metastatic potential of prostate cancer cells. Cancer Res 66: 7509-7515, 2006.

9. Liu R, Tian B, Gearing M, Hunter S, Ye K and Mao Z: Cdk5-mediated regulation of the PIKE-A-Akt pathway and glioblastoma cell invasion. Proc Natl Acad Sci USA 105: 7570-7575, 2008.

10. Demelash A, Rudrabhatla P, Pant HC, Wang X, Amin ND, McWhite CD, Naizhen X and Linnoila RI: Achaete-scute homologue-1 (ASH1) stimulates migration of lung cancer cells through Cdk5/p35 pathway. Mol Biol Cell 23: 2856-2866, 2012.

11. Feldmann G, Mishra A, Hong SM, Bisht S, Strock CJ, Ball DW, Goggins M, Maitra A and Nelkin BD: Inhibiting the cyclin-dependent kinase CDK5 blocks pancreatic cancer formation and progression through the suppression of Ras-Ral signaling. Cancer Res 70: 4460-4469, 2010.

12. Lin H, Chen MC, Chiu CY, Song YM and Lin SY: Cdk5 regulates STAT3 activation and cell proliferation in medullary thyroid carcinoma cells. J Biol Chem 282: 2776-2784, 2007.

13. Goodyear S and Sharma MC: Roscovitine regulates invasive breast cancer cell (MDA-MB231) proliferation and survival through cell cycle regulatory protein cdk5. Exp Mol Pathol 82: 25-32, 2007.

14. Liu JL, Wang XY, Huang BX, Zhu F, Zhang RG and Wu G: Expression of CDK5/p35 in resected patients with non-small cell lung cancer: relation to prognosis. Med Oncol 28: 673-678, 2011.

15. Jemal A, Murray T, Ward E, Samuels A, Tiwari RC, Ghafoor A, Feuer EJ and Thun MJ: Cancer Statistics, 2005. CA Cancer J Clin 55: 10-30, 2005.

16. Pozo K, Castro-Rivera E, Tan C, Plattner F, Schwach G, Siegl V, Meyer D, Guo A, Gundara J, Mettlach G, Richer E, et al: The role of Cdk5 in neuroendocrine thyroid cancer. Cancer Cell 24: 499-511, 2013.

17. Li D, Jia H, Zhang H, Lv M, Liu J, Zhang Y, Huang T and Huang B: TLR4 signaling induces the release of microparticles by tumor cells that regulate inflammatory cytokine IL- 6 of macrophages via microRNA let-7b. Oncoimmunology 1: 687-693, 2012.

18. Goodwin PR, Sasaki JM and Juo P: Cyclin-dependent kinase 5 regulates the polarized trafficking of neuropeptide-containing dense-core vesicles in Caenorhabditis elegans motor neurons. J Neurosci 32: 8158-8172, 2012. 